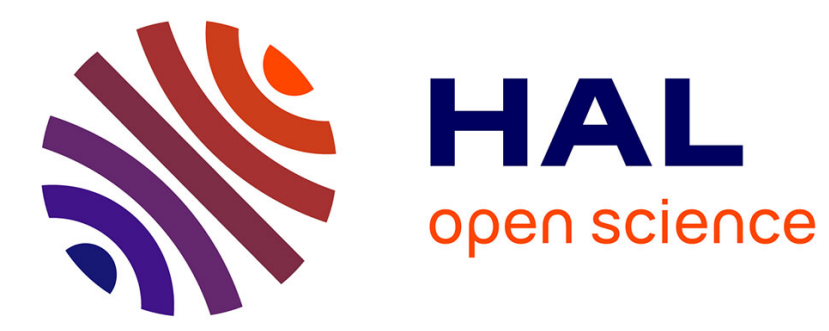

\title{
The role of the image phase in cardiac strain imaging
} Martino Alessandrini, Adrian Basarab, Mathieu de Craene, Maxime Sermesant, Hervé Liebgott, Olivier Bernard, Jan d'Hooge

\section{To cite this version:}

Martino Alessandrini, Adrian Basarab, Mathieu de Craene, Maxime Sermesant, Hervé Liebgott, et al.. The role of the image phase in cardiac strain imaging. EUSIPCO 2015 - 23rd European Signal Processing Conference, Aug 2015, Nice, France. pp.2796-2800, 10.1109/EUSIPCO.2015.7362894. hal-01265886v2

\section{HAL Id: hal-01265886 https://hal.science/hal-01265886v2}

Submitted on 26 Feb 2016

HAL is a multi-disciplinary open access archive for the deposit and dissemination of scientific research documents, whether they are published or not. The documents may come from teaching and research institutions in France or abroad, or from public or private research centers.
L'archive ouverte pluridisciplinaire HAL, est destinée au dépôt et à la diffusion de documents scientifiques de niveau recherche, publiés ou non, émanant des établissements d'enseignement et de recherche français ou étrangers, des laboratoires publics ou privés. 


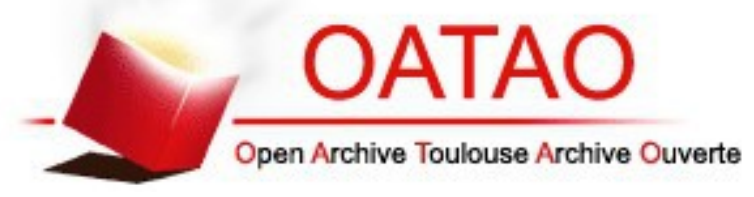

\title{
Open Archive TOULOUSE Archive Ouverte (OATAO)
}

OATAO is an open access repository that collects the work of Toulouse researchers and makes it freely available over the web where possible.

This is an author-deposited version published in : http://oatao.univ-toulouse.fr/ Eprints ID : 14906

To link to this article : DOI : 10.1109/EUSIPCO.2015.7362894

URL : http://dx.doi.org/10.1109/EUSIPCO.2015.7362894

\begin{abstract}
To cite this version : Alessandrini, Martino and Basarab, Adrian and De Craene, Mathieu and Sermesant, Maxime and Liebgott, Hervé and Bernard, Olivier and D'Hooge, Jan The role of the image phase in cardiac strain imaging. (2015) In: 23rd European Signal Processing Conference (EUSIPCO) 2015, 31 August 2015 - 4 September 2015 (Nice, France).
\end{abstract}

Any correspondance concerning this service should be sent to the repository administrator: staff-oatao@listes-diff.inp-toulouse.fr 


\title{
THE ROLE OF THE IMAGE PHASE IN CARDIAC STRAIN IMAGING
}

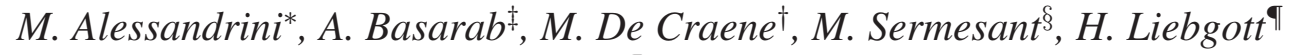 \\ O. Bernard ${ }^{\mathbb{N}}$, J. D’hooge* \\ *Cardiovascular Imaging and Dynamics, KU Leuven; \\ $\dagger$ Medisys, Philips Research Paris; \\ ‡ IRIT; CNRS UMR5505; 118 Route de Narbonne, F-31062, Toulouse cedex 9, France; \\ $\S$ Asclepios Project, INRIA; \\ ${ }^{\top}$ CREATIS; CNRS UMR5220; Inserm U1044; INSA-Lyon.
}

\begin{abstract}
This paper reviews our most recent contributions in the field of cardiac deformation imaging, which includes a motion estimation framework based on the conservation of the image phase over time and an open pipeline to benchmark algorithms for cardiac strain imaging in 2D and 3D ultrasound. The paper also shows an original evaluation of the proposed motion estimation technique based on the new benchmarking pipeline.
\end{abstract}

Index Terms - medical imaging, heart, deformation imaging, echocardiography, MRI, quality assurance, simulations.

\section{INTRODUCTION}

Evolving medical imaging technologies such as ultrasound (US) and magnetic resonance imaging (MRI) allow visualizing the cardiac muscle non-invasively with increasingly high spatial and temporal resolution. Unfortunately, the evaluation of cardiac motion still mainly relies on visual assessment and hence remains subjective and poorly reproducible between observers. The automated and reliable computation of quantitative indexes of cardiac deformation is still an open challenge which drives considerable research efforts. Our most recent achievements concerned two aspects of this problem:

A. Phase based motion estimation: A variety of techniques for the estimation of cardiac motion from medical images is available, which can be roughly subdivided between the families of block-matching, optical flow and elastic registration [1]. Regardless the family, most algorithms exploit the classical brightness constancy, assuming that a given portion of tissue generates the same image intensity over the full acquisition. Importantly, this assumption is often violated in medical imaging: in US the local brightness varies due to muscle deformation, rotation, changing angle between cardiac fibers and acoustic beams and (in 2D) out-of-plane motion; in MRI this is due to the inhomogeneity of the magnetic field; in tagged-MRI (tMRI) tags fade exponentially over time depending on the T1 relaxation time [2].

In this context, based on previous encouraging studies from other groups $[3,4]$, we concentrated on replacing brightness constancy with phase constancy. Image phase encodes information on the structure of the image while being independent on the local intensity. As such, this feature can be seen as an intrinsic signature of the tissue, insensitive to intensity variations due to the acquisition process. Hereto, we analyzed several definitions of image phase in function of their capability to encode relevant image features and to derive accurate motion estimators. They include the monogenic signal $[2,5,6]$, the structure multivector [7] and single-orthant signals [8].

B. Quality Assurance for deformation imaging: Despite the overwhelming availability of techniques for computation of cardiac deformation and strain, their widespread dissemination in clinical practice is still held back by the reported low reproducibility between different solutions. The concern is particularly actual for 2D and 3D US [9] and is partly due to the lack of a solid and open quality assurance framework. In this context, we have been active in the development of better benchmarking solutions based on the employment of realistic simulated datasets [1,10-12]. In particular, our latest contribution combines recent advances in the fields of electromechanical (E/M) modeling and ultrasound simulation to return synthetic echocardiograms virtually indistinguishable from real recordings [11]. The new framework represents a considerable leap forward with respect to existing solutions [13] and is completely open ${ }^{1}$ [11]. Originally developed for $3 \mathrm{D}$ datasets, the pipeline was recently extended to 2D [12].

The remainder of the paper proceeds as follows. Several definitions of image phase are reviewed in Sect. 2. The framework for phase based motion estimation, specialized for the monogenic signal, is presented in Sect. 3. The 2D benchmarking pipeline is described in Sect. 4. Sect. 5 summarizes

\footnotetext{
${ }^{1}$ https://team.inria.fr/asclepios/data/straus/
} 


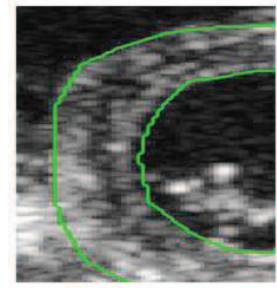

(a) US image

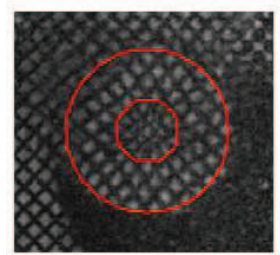

(d) Tagged MR

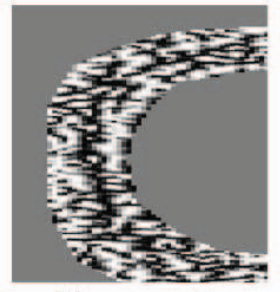

(b) monogenic

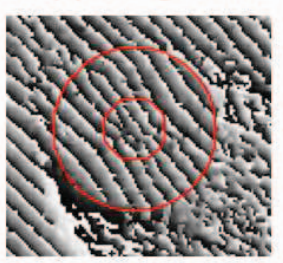

(e) Phase 1

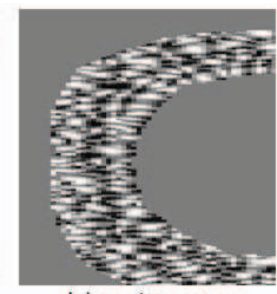

(c) multivector

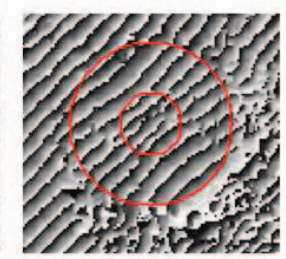

(f) Phase 2
Fig. 1. (a) test ultrasound image. (b) monogenic phase $\cos (\varphi)$. (c) phase from structure mutivector $\cos \left(\varphi_{1}\right)+\cos \left(\varphi_{2}\right)$. (d) test tagged MRI image. (e) first phase from structure multivector. (f) second phase from structure multivector. (a-c) Modified with permission from [7].

some of the obtained results in terms of motion estimation plus an original evaluation of the proposed algorithm on the new simulation pipeline.

\section{IMAGE PHASE}

Several definitions of image phase exist. The monogenic signal [14] extends the concept of analytic signal to images. It assumes an image $I(\mathbf{x})$ can be locally described in terms of 1D features (e.g. edges and ridges), according to the model:

$$
I(\mathbf{x})=A(\mathbf{x}) \cos (\varphi(\mathbf{x})), \quad \varphi(\mathbf{x})=2 \pi f_{0}(\mathbf{x}) \mathbf{n}^{T} \mathbf{x}
$$

being $A(\mathbf{x})$ the local amplitude, $\varphi(\mathbf{x})$ the local phase, $\mathbf{n}=$ $[\cos \theta, \sin \theta]$ the local orientation and $f_{0}(\mathbf{x})$ the local frequency. Amplitude encodes local image energy. Orientation and phase relate instead to the structure of the image and are independent on the amplitude. The three features are computed directly from the output to a bank of three quadrature filters (one even filter and two odd filters along $x$ and $y$ directions). When applied to US images, monogenic phase produces an intensity invariant representation of the typical speckle texture (cf. Fig. 1(a,b)).

The structure multivector [15] extends this definition by assuming the presence of two 1D orthogonal components, i.e.

$$
I(\mathbf{x})=\sum_{i=1}^{2} A_{i}(\mathbf{x}) \cos \left(2 \pi f_{0}(\mathbf{x}) \mathbf{n}_{i}^{T} \mathbf{x}\right)
$$

with $\mathbf{n}_{i}=\left[\cos \theta_{i}, \sin \theta_{i}\right]$ and $\theta_{2}=\theta_{1}+\pi / 2$. We showed in [7] that this formalism allows describing the speckle patterns of US images with finer detail as compared to the mono-

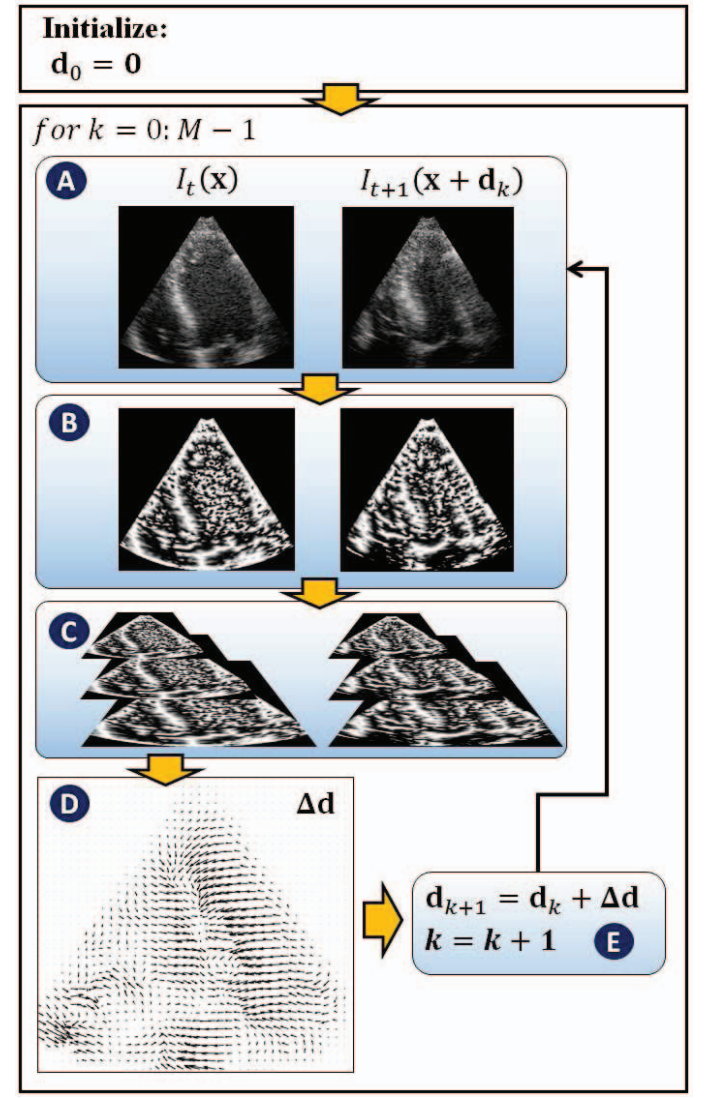

Fig. 2. Optical flow estimation. After initialization, the iterative optic flow computation proceeds as follows: two successive frames are loaded (A); monogenic features are then computed (B); the optimal window size for optic flow computation is optimized locally with a coarse-to-fine selection strategy $(\mathrm{C})$; the computed displacement field is used to increment the previous estimate (E) and subtracted to the second frame (A, right). Steps (A) through (E) are iterated $M$ times.

genic signal (cf. Fig. 1(c)). Moreover, the signal multivector is ideally adapted to encode the structure of tagged MRI images (cf. Fig. 1(d,f)): the two signal components in (2) capture the orthogonal tag directions, while the frequency $f_{0}$ relates to the tag spacing [6].

Finally, a phase definition based on single orthant analytic signals was adopted in [8]. The signal model is:

$$
I(x, y)=w_{s}(x, y) \cos \left(2 \pi f_{1} x\right) \cos \left(2 \pi f_{2} y\right)
$$

with $w_{s}(x, y)$ a low-pass 2-D window having its highest frequency lower than the frequency of the 2-D cosinus. The image is hence the result of two orthogonal monochromatic waves traveling along the coordinate axes. The tool was used to develop a motion estimator for US images with transverse oscillations (TO-US), i.e. in which the acquisition sequence is modified so to add oscillations in the direction perpendicular to the beam propagation. The two components relate then to the standard axial and the added lateral oscillation directions. 


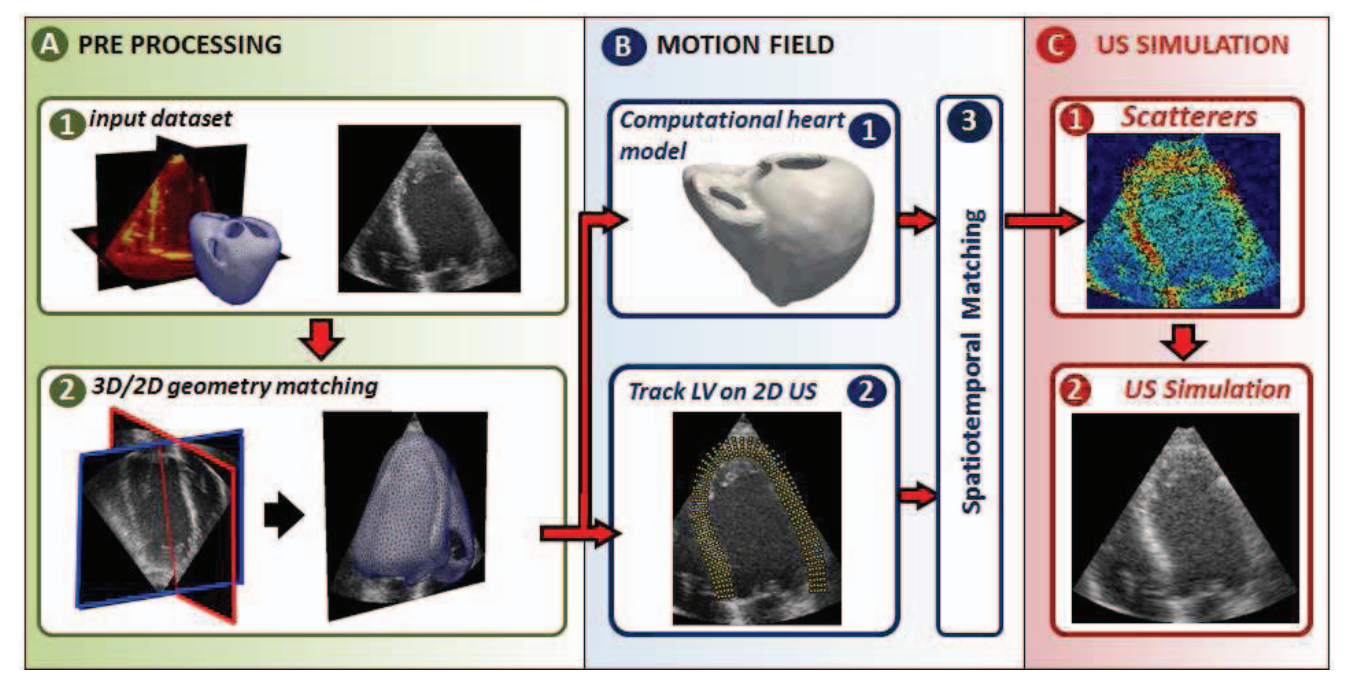

Fig. 3. Pipeline to generate realistic simulated echocardiographic sequences. Videos showing examples of simulated sequences can be found at http://bit.ly/1qmcFbt.

\section{PHASE BASED MOTION ESTIMATION}

The employed framework for motion estimation is summarized in Fig. 2. Following [2], the description in this section is specialized for the case of the monogenic phase. With minor modifications, the same work-flow was applied to the other definitions of image phase described in Sect. 2. For details please refer to the cited articles. The algorithm computes the displacement field $\mathbf{d}=[u, v]$ between two subsequent frames $I_{t}(\mathbf{x})$ and $I_{t+1}(\mathbf{x})$ of a sequence. This is done by translating the classical assumption on the conservation of the image intensity $I$ to the monogenic phase $\varphi$, i.e. $\varphi_{t+1}(\mathbf{x})=\varphi_{t}(\mathbf{x}-\mathbf{d})$.

By assuming small displacements, the equation can be linearized and the motion estimation problem can be solved in the least squares sense over an image patch $\Omega$ by minimizing:

$$
E(u, v)=\int_{\Omega} w \cdot\left(\varphi_{x} u+\varphi_{y} v+\varphi_{t}\right)^{2} d x d y
$$

where $w$ is a weighting function penalizing pixels close to the boundary of the patch. We assume a local affine model for the velocity field within each patch:

$$
\left(\begin{array}{l}
u \\
v
\end{array}\right)=\left(\begin{array}{ll}
u_{x} & u_{y} \\
v_{x} & v_{y}
\end{array}\right) \cdot\left(\begin{array}{c}
x-x_{0} \\
y-y_{0}
\end{array}\right)+\left(\begin{array}{l}
u_{0} \\
v_{0}
\end{array}\right),
$$

being $u_{0}$ and $v_{0}$ the velocity components at the center $\left(x_{0}, y_{0}\right)$ of the local patch. Plugging (5) in (4) and minimizing, leads to the solution of a linear system of equations $\mathbf{A d}=\mathbf{b}$ which gives the desired motion estimate. Entries of $\mathbf{A}$ and $\mathbf{b}$ are the cross products of spatial and temporal derivatives of the image phase. Interestingly, these quantities can be directly computed from the output of the quadrature filters thereby avoiding phase wrapping issues [2].

By expressing $w$ as the tensor product of B-spline functions, a computationally efficient coarse-to-fine strategy can be put in place which locally optimizes the size of the local patch in order to give the most consistent velocity estimates, as measured by the residual $\|\mathbf{A d}-\mathbf{b}\|_{2}$ [16]. This solution allows optimizing the compromise between having robust estimates (requiring larger windows) and a detailed displacement field (requiring smaller windows). Finally, in order to deal with large motions, the displacement estimate is iteratively refined in a pyramidal scheme: a coarse representation of the images is used for an initial guess, the motion estimate is then progressively refined on images at higher resolution by using the previous result as initialization.

\section{EVALUATION WORKFLOW}

The overall workflow is summarized in Fig. 3. Briefly, the proposed solution combines an advanced E/M heart model to determine the synthetic motion with a real ultrasound recording working as a template for speckle texture. The output is a simulated sequence visually similar to the template recording but with fully controlled cardiac motion. The known motion can be used to benchmark algorithm for cardiac deformation/strain imaging. The pipeline is described in detail in [12].

The first step (A) is to obtain a 3D segmentation of a 2D acquisition. A.1: On the same healthy volunteer, one 3D and one 2D (apical four chamber in Fig. 3) scan are recorded (GE Vivid 7 system, MS4 probe). From the first frame of the 3D scan right and left ventricles are segmented manually. A.2: The first frame of the 2D scan is then manually registered to the first frame of the 3D volume by using anatomical landmarks (apex and mitral annulus). The alignment also places the $2 \mathrm{D}$ sequence and the 3D cardiac anatomy in the same space. The synthetic motion field is then computed (B). B.1: The E/M heart model in [17] is applied to the $3 \mathrm{D}$ anatomy to generate one full cardiac cycle. By varying the parameters of 

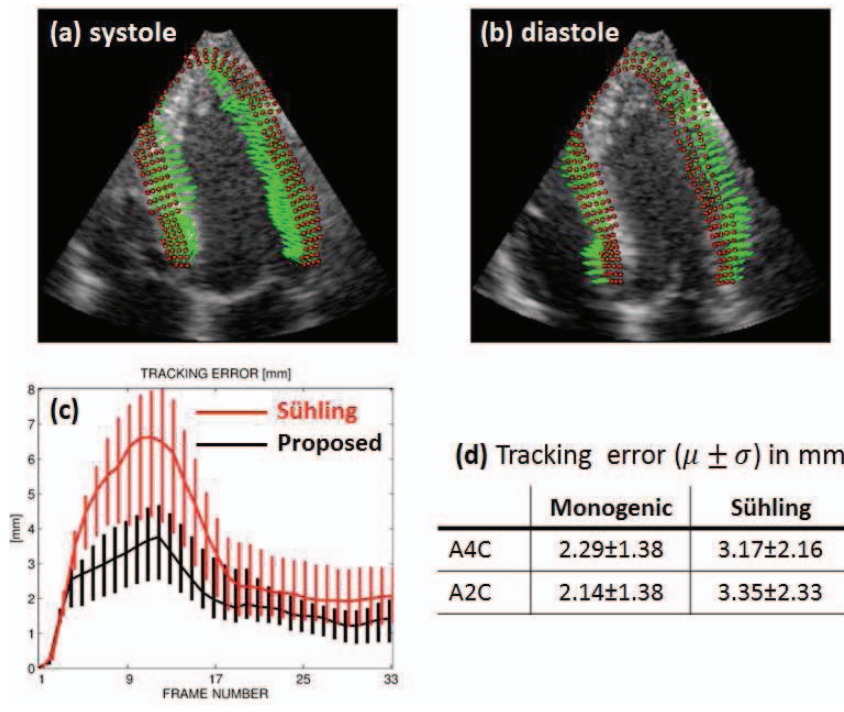

(d) Tracking error $(\mu \pm \sigma)$ in $\mathrm{mm}$

\begin{tabular}{l|c|c} 
& Monogenic & Sühling \\
\hline A4C & $2.29 \pm 1.38$ & $3.17 \pm 2.16$ \\
\hline A2C & $2.14 \pm 1.38$ & $3.35 \pm 2.33$ \\
\hline
\end{tabular}

Fig. 4. Estimated motion field and corresponding tracked position of test points for a systolic (a) and a diastolic (b) frame of the simulated A4C sequence. Note how the motion field reflects the expected contraction and relaxation of the cardiac muscle. In (c) the tracking error for the $\mathrm{A} 2 \mathrm{C}$ sequence. Bold time curves report median errors while vertical segment denote 25 -th and 75 -th percentile values. In (c) the average tracking errors for the two synthetic sequences employed.

the E/M model locally several pathological conditions can be simulated. B.2: The elastic registration technique in [18] is applied to cancel the motion in the 2D recording. B.3: From (B.1) and (B.2) a transform is computed to match in space and time the 3D E/M simulation with the $2 \mathrm{D}$ recording. Hereto, thin plate splines (TPS) parameterized by nodes coordinates of 3D E/M meshes (B.1) and of the 2D tracking (B.2) are used. The final step (C) is the ultrasound simulation. C.1: A cloud of point scatterers is defined to mimic the response of cardiac tissue to ultrasound stimulation. Myocardial scatterers are displaced according to the E/M simulation. Scattering amplitude is sampled from the $2 \mathrm{D}$ recording by using the previously computed spatiotemporal matching (B3). C.2: The scatter map is set as input of a fast US [19] to simulate the images.

\section{RESULTS}

\subsection{Previous Results}

The monogenic optical flow algorithm was evaluated and contrasted against state of the art solutions on synthetic images from several modalities: 2D echocardiography in [2], $3 \mathrm{D}$ echocardiography in [5] and tagged-MRI in [2,6]. On 2D US, the algorithm outperformed its counterpart exploiting conservation of image intensity [16], moreover, both on 2D US and 2D tagged-MRI, it was more accurate than other existing phase based solutions. On 3D US, the algorithm was competitive with other solutions from leading teams from academia and industry, and showed a particularly favorable compromise between accuracy and speed. The technique based on the structure multivector showed to be more accurate than the monogenic optical flow algorithm in an initial evaluation on synthetic 2D US recordings [7] and hence deserves further testing. Moreover, the possibility to use the signal multivector to encode relevant structural features of tagged MRI images (cf. Fig. 1) also requires more accurate investigation. Finally, the technique based on single orthant signals on TO-US images was proven more accurate than traditional block matching [8], which represents the current implementation on commercial systems.

\subsection{Results on the new evaluation pipeline}

The pipeline in Sect. 4 was used to contrast the proposed algorithm in Sect. 3 against [16]. The technique employs standard brightness constancy in a work-flow similar to the one in Fig. 2, with the exception of block $\mathbf{B}$. Hereto, we simulated 2 sequences: one apical 2 chamber (A2C) and one apical 4 chamber (A4C) from an healthy heart. As in [12], accuracy was computed from a set of seed points sampling the LV myocardium regularly in the longitudinal and radial directions (cf. Fig. 4). The points were then propagated by using either the ground truth motion or the result of the tracking algorithm. For each point, tracking error was determined by the euclidean distance between the ground truth and the estimated position. For both algorithms, parameters were optimized to minimize the average tracking error on the healthy A4C simulation. The results are summarized in Fig. 4.

The proposed algorithm returns the most accurate (smallest average error) and consistent (lower limits of agreement) displacement estimates. Moreover, the estimated displacement field reflects qualitatively the expected contraction/relaxation patterns of the cardiac muscle during systole and diastole. This additional evaluation confirms the interest of the image phase to replace or complement image intensity for motion tracking.

\section{CONCLUSIONS}

We described our most recent advances in the field of cardiac deformation/strain imaging. We first introduced the image phase as a relevant feature for motion tracking with important conceptual advantages over the traditional image intensity. Hereto, we also described a phase-based framework for cardiac motion estimation. A novel pipeline for the quality assurance of algorithms for deformation/strain imaging on 2D and 3D ultrasound was also described. We concluded by summarizing our previously obtained results and by presenting an original evaluation of the proposed motion estimator on the newly introduced benchmarking pipeline.

\section{REFERENCES}


[1] M. De Craene, S. Marchesseau, and B. Heyde et al., "3D strain assessment in ultrasound (straus): A synthetic comparison of five tracking methodologies," IEEE TMI, vol. 32, no. 9, pp. 1632-1646, 2013.

[2] M. Alessandrini, A. Basarab, H. Liebgott, and O. Bernard, "Myocardial motion estimation from medical images using the monogenic signal," IEEE TIP, vol. 22, no. 3, pp. 1084-1095, 2013.

[3] K. Rajpoot, V. Grau, and J.A. Noble, "Local-phase based $3 \mathrm{~d}$ boundary detection using monogenic signal and its application to real-time 3-d echocardiography images," in Proc. of IEEE ISBI, June 2009, pp. 783786.

[4] A. Belaid, D. Boukerroui, Y. Maingourd, and J.-F. Lerallut, "Phase-based level set segmentation of ultrasound images," IEEE Trans Inf Technol Biomed, vol. 15, no. 1, pp. 138-147, Jan 2011.

[5] M. Alessandrini, H. Liebgott, D. Barbosa, and O. Bernard, "Monogenic phase based optical flow computation for myocardial motion analysis in 3d echocardiography," in Proc. of MICCAI, vol. 7746, pp. 159168. 2013.

[6] M. Alessandrini, H. Liebgott, A. Basarab, P. Clarysse, and O. Bernard, "Monogenic signal for cardiac motion analysis from tagged magnetic resonance image sequences," in Proc. of CinC, Sept 2012, pp. 685-688.

[7] M. Alessandrini, H. Liebgott, D. Friboulet, and O. Bernard, "Cardiac motion assessment from echocardiographic image sequences by means of the structure multivector," in Proc. of IEEE IUS, Oct 2013, pp. 10981101.

[8] M. Alessandrini, A. Basarab, L. Boussel, X. Guo, A. Serusclat, D. Friboulet, D. Kouame, O. Bernard, and H. Liebgott, "A new technique for the estimation of cardiac motion in echocardiography based on transverse oscillations: A preliminary evaluation in silico and a feasibility demonstration in vivo," IEEE TMI, vol. 33, no. 5, pp. 1148-1162, May 2014.

[9] Victor Mor-Avi, Roberto M. Lang, and Luigi P. Badano et al., "Current and evolving echocardiographic techniques for the quantitative evaluation of cardiac mechanics: Ase/eae consensus statement on methodology and indications: Endorsed by the japanese society of echocardiography," JASE, vol. 24, no. 3, pp. 277 - 313, 2011.

[10] M. Alessandrini, H. Liebgott, D. Friboulet, and O. Bernard, "Simulation of realistic echocardiographic sequences for ground-truth validation of motion estimation," in IEEE Proc. of ICIP, Sept 2012, pp. 2329-2332.

[11] M. Alessandrini, M. De Craene, O. Bernard, S. GiffardRoisin, P. Allain, J. Weese, E. Saloux, H. Delingette,
M. Sermesant, and J. D'hooge, "A pipeline for the generation of realistic $3 \mathrm{~d}$ synthetic echocardiographic sequences: Methodology and open-access database.," IEEE TMI, in press.

[12] M. Alessandrini, B. Heyde, S. Giffard-Roisin, H. Delingette, M. Sermesant, P. Allain, O. Bernard, M. De Craene, and J. D'hooge, "Generation of ultra-realistic synthetic echocardiographic sequences to facilitate standardization of deformation imaging," in Proc. of IEEE ISBI, 2015, accepted.

[13] A. Elen, Hon Fai Choi, and D. Loeckx et al., "Threedimensional cardiac strain estimation using spatio temporal elastic registration of ultrasound images: A feasibility study," IEEE TMI, vol. 27, no. 11, pp. 1580-1591, 2008.

[14] M. Felsberg and G. Sommer, "The monogenic signal," IEEE TIP, vol. 49, no. 12, pp. 3136-3144, Dec 2001.

[15] Michael Felsberg and Gerald Sommer, "Image features based on a new approach to $2 \mathrm{~d}$ rotation invariant quadrature filters," in Proc. of ECCV, vol. 2350 of Lecture Notes in Computer Science, pp. 369-383. Springer Berlin Heidelberg, 2002.

[16] M. Sühling, M. Arigovindan, C. Jansen, P. Hunziker, and M. Unser, "Myocardial motion analysis from bmode echocardiograms," IEE TIP, vol. 14, no. 4, pp. 525-536, 2005.

[17] S. Marchesseau, H. Delingette, M. Sermesant, M. Sorine, K. Rhode, S.G. Duckett, C.A. Rinaldi, R. Razavi, and N. Ayache, "Preliminary specificity study of the Bestel-Clement-Sorine electromechanical model of the heart using parameter calibration from medical images," J Mech Behav Biomed Mater, vol. 20, pp. 259-271, 2013.

[18] B. Heyde, R. Jasaityte, D. Barbosa, V. Robesyn, S. Bouchez, P. Wouters, F. Maes, P. Claus, and J. D'hooge, "Elastic image registration versus speckle tracking for 2-d myocardial motion estimation: A direct comparison in vivo," IEEE TMI, vol. 32, no. 2, pp. 449-459, Feb 2013.

[19] Hang Gao, Hon Fai Choi, P. Claus, S. Boonen, S. Jaecques, G.H. Van Lenthe, G. Van der Perre, W. Lauriks, and J. D'hooge, "A fast convolution-based methodology to simulate 2D/3D cardiac ultrasound images," IEEE TUFFC, vol. 56, no. 2, pp. 404-409, 2009. 